European Association for the

Development of Renewable Energies,

Environment and Power Quality
International Conference on Renewable Energies and Power Quality (ICREPQ’09)

Valencia (Spain), 15th to 17th April, 2009

\title{
The Fundamental Particle and Energy Quanta of Dark Matter and Dark Energy: Boltzmann Particles and Utilization its Energy
}

\author{
Murad Shibli, Visiting Assistant Professor \\ Mechanical Engineering Department \\ College of Engineering, United Arab Emirates University, Al-Ain, United Arab Emirates \\ P.O. Box 17555, Cell: +971-5-9328-707 \\ malshibli@uaeu.ac.ae
}

\begin{abstract}
This paper presents what could be the most fundamental particle and quanta of dark matter and dark energy and its characteristics. Recent observations have shown that visible matter contributes only to about $4 \%$ of the universe total energy density, meanwhile, dark matter and dark energy contributes to $26 \%$ and $70 \%$ of the universe total energy, respectively. This paper is a continuation of previous published work at ICREPQ'08 and ICREPQ'07 which was based on the assumption that dark energy and dark matter are identical and behave as an ideal gas. Furthermore, based on Boltzmann constant and NASA's Cosmic Microwave Background Explorer (CMB) which estimated that the sky has a temperature close to 2.7251 Kelvin, then the equivalent mass and energy of fundamental particle of the dark matter/dark energy is determined. It is found that this candidate particle has an equivalent mass of $4.2141 \times 10^{-40} \mathrm{Kg}$ which is equivalent to $3.7674 \times 10^{-23} \mathrm{~J}$. Since this value has the same order of Boltzmann constant $K_{B}=1.38 \times 10^{-23} \mathrm{~J} / \mathrm{K}$, then this particle is called Boltzmann particle. Furthermore, considering the lowest temperature in nature at Boomerang nebula which is 1 Kelvin, then the dark matter should be exactly equivalent to Boltzmann constant. Boltzmann particle could be the most fundamental and lightest particle in Nature. Moreover, assuming a uniform space dark energy/dark matter density, then the critical temperature at which the dark matter has a unity entity per volume is determined as $34.983 \times 10^{12} \mathrm{~K}$. This analysis shows that the dark matter is a preferably a cold matter since its volumetric density is inversely proportional to the absolute temperature of the space.
\end{abstract}

Keywords- Dark Energy, Dark Matter, Equation of State, Boltzmann Constant, Einstine's Cosmological Constant.

\section{PRELIMINARY: THE EQUATION OF STATE OF DARK ENERGY AND DARK MATTER: THE UNIFIED ENTITY}

This equation relates the pressure $P$, temperature $T$ and the volume $V$ of a substance behaves as an ideal gas [2], that is $P V=m R T$

As it can be seen easily that equation (1) represents the energy associated with an ideal gas at given pressure $P$, temperature $T$ and the volume $V$, that is

$P V=m R T=E$
Note that both sides of the equation has the units of energy (work done by pressure $P$ ). Assume now that dark energy behaves like an ideal gas with a negative pressure $(-P)$ that causes the universe to expand with a total volume $V$, then by dividing both side of the equation of state (5) by $V$, then

$P=\frac{m}{V} R T=\frac{E}{V}$

Defining the mass density as $\rho_{m}=\frac{m}{V}$ and energy density as

$\rho_{E}=\frac{E}{V}$, equation (3) yields to

$P=\rho_{m} R T=\rho_{E}$

Now by taking the ratio between the mass density and energy density then

$\frac{\rho_{E}}{\rho_{m}}=R T$

It can be concluded that the ratio between the mass density and energy density are proportional to the product of the temperature $T$ and dark energy-dark matter constant $R$ (known as Universal gas constant). It is worth to mention that NASA's Cosmic Microwave Background Explorer (CMB) in 1992 estimated that the sky has a temperature close to 2.7251Kelvin. Moreover, the Wilkinson Microwave Anisotropy Probe (WMAP) in 2003 has made a map of the temperature fluctuations of the CMB with more accuracy [11].

The Boltzmann constant $K_{B}$ is a physical constant that relates temperature to microscopic energy. $\mathrm{K}_{\mathrm{B}}=R / N_{A}$, where $\quad N_{A}$ is the Avogadro Number. $\mathrm{K}_{\mathrm{B}}=1.38 \times 10^{-23} \mathrm{~J} / \mathrm{K}$ The numerical value of $\mathrm{K}_{\mathrm{B}}$ measures the conversion factor for mapping from this microscopic energy $E$ to the macroscopically-derived temperature scale.

The ideal gas law can now be expressed in terms of Boltzmann constant such that 
$P V=N K_{B} T$

where $N$ is the actual number of entities (particles). Now dividing both sides of (10) by volume to get the energy density then

$$
P=\frac{N}{V} K_{B} T=\rho_{N} K_{B} T=\rho_{E}
$$

This shows that the ratio between the energy density and the entities density is proportional to the absolute temperature times the Boltzmann constant.

\section{BoltzMAnN PARTiCles}

Based on astronomical observations that the average density of dark matter and dark energy is approximately $10^{-26} \mathrm{Kg} / \mathrm{m}^{3}$ and based on previous published work [12] that the density of dark matter is $0.54 \times 10^{-26} \mathrm{Kg} / \mathrm{m}^{3}$ which is equivalent to $4.8277 \times 10^{-10} \mathrm{~J} / \mathrm{m}^{3}$. Now benefiting from (7) at CMB temperature $\mathrm{T}=2.73 \mathrm{~K}$, then

$$
\rho_{N}=12.81 \times 10^{12} \text { entities } / \mathrm{m}^{3}
$$

Since $\rho_{N}=12.81 \times 10^{12}$ entities $/ \mathrm{m}^{3}$ is corresponding to $0.54 \times 10^{-26} \mathrm{Kg} / \mathrm{m}^{3}$, then each entity has a mass of $0.54 \times 10^{-26} / 12.81 \times 10^{12}=4.2141 \times 10^{-40} \mathrm{Kg}$. The equivalent energy of this particle is $3.7674 \times 10^{-23} \mathrm{~J}$. Furthermore, considering the lowest temperature in nature at Boomerang nebula which is 1 Kelvin, then the dark matter should be exactly equivalent to Boltzmann constant. Since the particle equivalent energy has the same order of Boltzmann constant, then it is called Boltzmann particle. As it can be seen, the mass of the electron is much heavier than this Boltzmann particle by 2.159 Billion times. Furthermore, $\rho_{N}$ is unity when the temperature $\mathrm{T}$ is equal to $34.983 \times 10^{12} \mathrm{~K}$. This temperature value is called the critical temperature. It is estimated that at 100 microseconds after the Big Bang the temperature was $10 \mathrm{TK}$. At 3-5 TK proton-antiproton reactions occur. If the density of dark matter/dark energy is uniform, homogeneous and constant through the universe, and since the density is at the same order of the proton-nitron, then it is very possible that dark energy/dark matter is converted into quarks at this critical temperature.

\section{Proposed Boltzmann Particle, $B$}

$\begin{array}{lcc}m_{B} & 4.2141 \times 10^{-40} & \mathrm{~kg} \\ & 2.5386 \times 10^{-13} & u \\ m_{B} c^{2} & 3.7674 \times 10^{-23} & \mathrm{~J} \\ & 0.022695 & \mu \mathrm{eV}\end{array}$

\section{UTILIZATION OF DARK MATTER/DRAK ENERGY}

Since dark matter/dark energy behaves like an ideal fluid and there density are affected by the space temperature then utilization of its energy can be achieved at high temperatures such as Fermi melting point of quarks into quark-gluon plasma $\left(0.5-1.2 \times 10^{12} \mathrm{~K}\right)$ or nuclear fusion $\left(1-100 \times 10^{9} \mathrm{~K}\right)$. Furthermore, $\rho_{N}$ is unity when the temperature $\mathrm{T}$ is equal to $34.983 \times 10^{12} \mathrm{~K}$. This temperature value is called the critical temperature. It is estimated that at 100 microseconds after the Big Bang the temperature was 10 TK. At 3-5 TK protonantiproton reactions occur. If the density of dark matter/dark energy is uniform, homogeneous and constant through the universe, and since the density is at the same order of the proton-nitron, then it is very possible that dark enrgy/dark matter is converted into quarks at this critical tmaprature.

\section{CONCLUISON}

This paper presents what could be the most fundamental particle and quanta of dark matter and dark energy and its characteristics. It is found that this candidate particle has an equivalent mass of $4.2141 \times 10^{-40} \mathrm{Kg}$ which is equivalent to $3.7674 \times 10^{-23} \mathrm{~J}$. Since this value has the same order of Boltzmann constant $\mathrm{K}_{\mathrm{B}}=1.38 \times 10^{-23} \mathrm{~J} / \mathrm{K}$, then this particle is called Boltzmann particle. Boltzmann particle could be the most fundamental and lightest particle in Nature. Moreover, the critical temperature at which the dark matter has a unity entity per volume is determined as $34.983 \times 10^{12} \mathrm{~K}$. This analysis shows that the dark matter is a preferably a cold matter since its volumetric density is inversely proportional to the absolute temperature of the space. Utilization of its energy can be achieved at high temperatures such as Fermi melting point of quarks into quark-gluon plasma $\left(0.5-1.2 \times 10^{12} \mathrm{~K}\right.$ ) or nuclear fusion $\left(1-100 \times 10^{9} \mathrm{~K}\right)$.

\section{REFERENCES}

[1] A. Einstein, "The Foundation of the General Theory of Relativity," pp., 146-200, [The collected papers of Einstein, English Translation Edited by A.J. Kox, M.J. Klien, and R. Schulmann, Volume 6, Princeton University Press, 1997], The Berlin Years Writings, 1914-1917.

[2] Y. A. Cengel, M. A. Boles, Thermodynamics: an Engineering Approach, Fifth Edition, McGraw Hill, 2006.

[3] S. M. Carroll, "The Cosmological Constant" Living Reviews of Relativity, 2001, retrieved on 2006.

[4] M. Shibli, "The Foundation of the Fourth Law of Thermodynamics: Dark Energy and its Nature: Can Dark Energy be Generated?," International Conference on Renewable Energies and Power Quality (ICREPQ'07), European Association for the Development of Renewable Energies, Environment and Power Quality, Full Script www.icrepq.com, http://www.icrepq.com/icrepq07/286-shibli.pdf.Seville,Spain, March $26^{\text {th }}-28^{\text {th }}, 2007$.

[5] M. Shibli, "The Equation of State of Dark Energy and Dark Matter: Boltzmann Constant and the Unified Entity: Utilization of Space Energy," (ICREPQ'08), , Full Script http://www.icrepq.com/icrepq-08/461_Shibli.pdf. Spain on March 12-14, 2008.

[6] M. Shibli, "The Foundation of the Theory of Dark Energy," IEEE International Conference on Space Technology (ICST), Istanbul, Turky, June $14^{\text {th }}-16^{\text {th }}, 2007$.

[7] M. S. Turner, "Dark Matter and Dark Energy in the Universe," The Third Astro. Symposium: The Galactic Halo ASP Conference Series, Vol. 666, 1999. 